NASZA DERMATOLOGIA Online OUR DERMATOLOGY Online

Source of Support: Nil

Competing Interests: None

\section{A CASE OF SUBCUTANEOUS PHAEOHYPHOMYCOSIS CAUSED BY EXSEROHILUM SPECIES IN AN IMMUNOCOMPROMISED PATIENT}

\author{
Koppada Rajasekhar, Anaparthy Usharani, \\ Nirupama Padmaja Bondili, Ratna Harika Dusi, \\ Perala Balamurali Krishna
}

Department of Microbiology, Siddhartha Medical College, Vijayawada, India

Corresponding author: Prof. Anaparthy Usharani

usharani.anaparthy@gmail.com

\begin{abstract}
Phaeohyphomycoses are rare fungal infections, caused by dematiaceous fungi, manifested as cutaneous and subcutaneous infections, meningitis, sinusitis, keratitis, osteomyelitis and disseminated infection.

This is a case report of a 45year old immuno compromised female on ART (Anti Retroviral therapy) presented with fever and generalized nodular lesions draining pus on face, hands, axilla, groin and labia majora since one month. Biopsy of the subcutaneous nodule on the lateral aspect of the thigh revealed septate fungal hyphae on $10 \% \mathrm{KOH}(10 \%$ Potassium Hydroxide) mount. Fungal culture of the biopsy material on SDA (Sabouraud's Dextrose Agar) at $25^{\circ} \mathrm{C}$ showed cotton wooly, dark gray to olivaceous black growth with black reverse and identified as dematiaceous fungi belonging to Exserohilum species by microscopy. The patient was put on Itraconazole 200mg BD in combination with Terbinafine $250 \mathrm{mg}$ BD for which she responded with healing of pustular lesions in two weeks and complete remission in two months.
\end{abstract}

Key words: subcutaneous nodules; Exserohilum species; Phaeohyphomycosis

Key message: The increase in the fungal infections may be related to a heightened awareness of these infection in humans and improved diagnostictechniques. Inspiteofavailabilityofbroadspectrumantifungalagentsthefungalinfectionsarecausing disasters, itisimperativetoinvestigate and pinpoint the causative agents in all infections to contribute data for further studies which will ultimately give an opportunity for better prevention and care.

Cite this article:

Koppada Rajasekhar, Anaparthy Usharani, Nirupama Padmaja Bondili, Ratna Harika Dusi, Perala Balamurali Krishna: A case of subcutaneous phaeohyphomycosis caused by Exserohilum species in an immunocompromised patient. Our Dermatol Online. 2013; 4(4): 498-500.

\section{Introduction}

Very few cases of subcutaneous phaeohyphomycosis caused by Exerohilum species were reported till date. On 17th October 2012 in the journal "Annals of Internal Medicine" there was an article reporting about an outbreak of fulminant meningitis caused by Exserohilum rostratum after receiving spinal epidural injections of methylprednisolone contaminated with Exserohilum rostratum causing loss of lives in USA [1]. From India very few cases of cutaneous phaeohyphomycosis have been published among which there is a case of cutaneous phaeohyphomycosis caused by Exserohilum rostratum in a 40 year old woman that presented to the Department of Biological sciences, Rani Durgavati University, Jabalpur, India in July 1995 with a 3 year history of a cutaneous infection of the upper anterior aspect of the forearm [2].
The phaeoid fungi are a curious group of organisms capable of causing a wide array of clinical pathologies ranging from the superficial to the deep seated infections. As a cosmopolitan disease, phaeohyphomycosis mainly afflicts adults among whom many are immunocompromised. Underlying conditions that contribute to infection by dematiaceous fungi include tuberculosis, diabetes, cancer, tissue transplantation, and use of corticosteroids or other immunosuppressive drugs, surgery, and a number of other conditions including acquired immunodeficiency syndrome (AIDS)due to the human immunodeficiency virus (HIV) [3].

The genus Exserohilum was established by Leonard and Suggs. It belongs to the Kingdom: Fungi, Phylum: Ascomycota, Class: Euascomycetes, Order: Pleosporales, Family: Pleosporaceae, Genus: Exserohilum. 
The three closely related genera Bipolaris, Drechsleria and Exserohilum are distinguished on the basis of such characters as conidial shape and size, hilar morphology, origin of the germ tubes from the basal or other conidial cells, and the location and sequence of the conidial septa. The only Exserohilum species known as etiologic agents of phaeohyphomycosis in humans and animals are E.rostratum, E.longirostratum and E.meginnisii $[4,5]$. An aggressive angioinvasive nature and the ability to grow at $400 \mathrm{C}$ indicate their potential to be neurotropic pathogens [1].

\section{Case Report}

A 45 year old immunocompromised female from a low socioeconomic background from Vijayawada presented with fever and generalized nodular lesions draining pus on face, hands, axilla, groin and labia majora since one month. No history of arthralgia, decreased sensations or any other systemic manifestations; Figure 1 showing the pustular lesions.

She was on Anti Retroviral Therapy since 4 years with an initial CD4+ count of 50 cells/ $\mu$ at the time of HIV diagnosis, which has improved to 500 cells/ $\mu \mathrm{l}$ at the time of presentation with the present complaints. The symptoms started with fever and upper respiratory infection for which she received antibiotics and after 15 days she developed a generalized pustular eruption for which she was also prescribeded antibiotics irregularly by a local practitioner. The patient did not show improvement and was referred to Government General Hospital, Vijayawada for further care.

On examination there were multiple bilateral generalized erythematous tender nodular lesions draining pus on face, hands, axilla, groin \& labia majora. Routine investigations like complete haemogram, LFT (Liver function tests), RPR (Rapid Plasma Reagin) were done and results were within normal limits. FBS -210mg/dl, PPBS -270mg/dl (Fasting blood sugar and Post prandial blood sugar levels are slightly out of range but no history of Diabetes or therapy with hypoglycemic drugs), ESR-46mm, CRP (C- reactive protein) - positive, ASO ( Anti steptolysin-O titre) - negative, SSS (Slit skin smears) are negative for Lepra bacilli. Biopsy of subcutaneous nodule on the lateral aspect of the thigh was sent to Department of Microbiology, for mycological diagnosis suspecting phaeohyphomycosis.

Mycological study and diagnosis:

$10 \% \mathrm{KOH}$ mount of the biopsy material revealed the presence

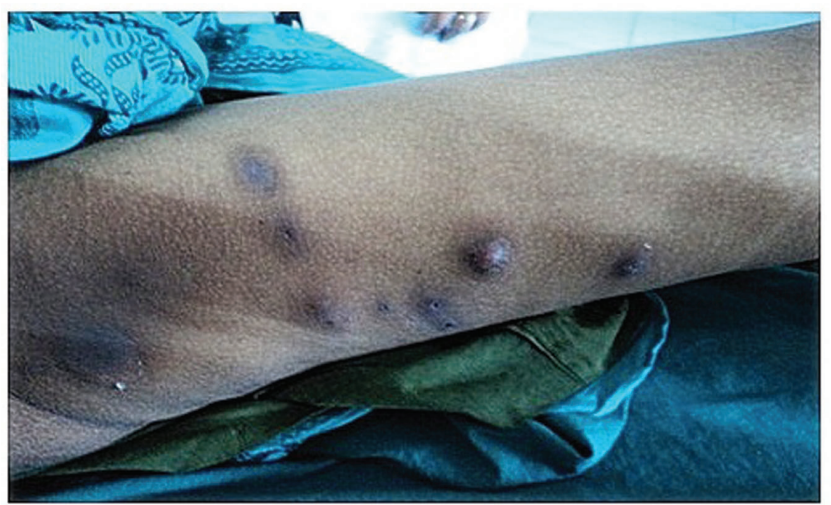

Figure 1. Subcutaneous nodules draining pus. of fungal elements showing slightly brown coloured hyphae with septations indicating pheoid fungi.

The biopsy material was inoculated directly onto Sabouraud's Dextrose Agar (SDA) and incubated at $25^{\circ} \mathrm{C}$. After 3 days of incubation cottony white growth appeared which turned to dark grey on further incubation and then turned to olivaceous black with a black reverse. Figure 2 showing the growth of Exserohilum spp. on the Sabouraud's Dextrose Agar slant.

Lactophenol Cotton Blue (LPCB) mount of the growth showed phaeoid septate branching hyphae with conidia. Conidia were ellipsoidal, distoseptate and had a protruding and truncate hilum. The fungus was identified as Exserohilum species based on the morphology of the conidia. Slide culture was done for further confirmation. Figure 3 showing the conidia of Exserohilum spp. in a LPCB mount.

The patient received treatment with Itraconazole $200 \mathrm{mg} B D$ in combination with Terbinafine $250 \mathrm{mg}$ BD for which she responded with healing of pustular lesions in two weeks. She continued this treatment for 2 months. Patient is on ART with marked improvement in general condition and complete remission of the pustular lesions. Figure 4 showing the healed pustular lesions after the antifungal treatment.

\section{Discussion}

Exserohilum may infect both immunocompromised and immunocompetent hosts with variable clinical manifestations, ranging from cutaneous infections to fulminant disseminated disease. During the last decades there has been an increase in the number of reported cases of Exserohilum infection. The increase may also be related to a heightened awareness of this infection in humans and improved diagnostic techniques. According to the review of the literature published by Adler A et al., there were 33 cases of Exserohilum infection, of which 23 were reported since 1993. Most occurred in regions with hot climates, such as India, Israel, and the Southern USA. Impaired immunity was present in the majority of patients with invasive and skin infections, whereas local trauma and atopy were the predisposing factors in those with corneal infections and allergic fungal sinusitis, respectively. Surgical debridement was the principal mode of therapy for allergic fungal sinusitis. Amphotericin B was the initial single antifungal agent used in all cases of invasive disease. The response rate was low but improved with addition of triazole agents.
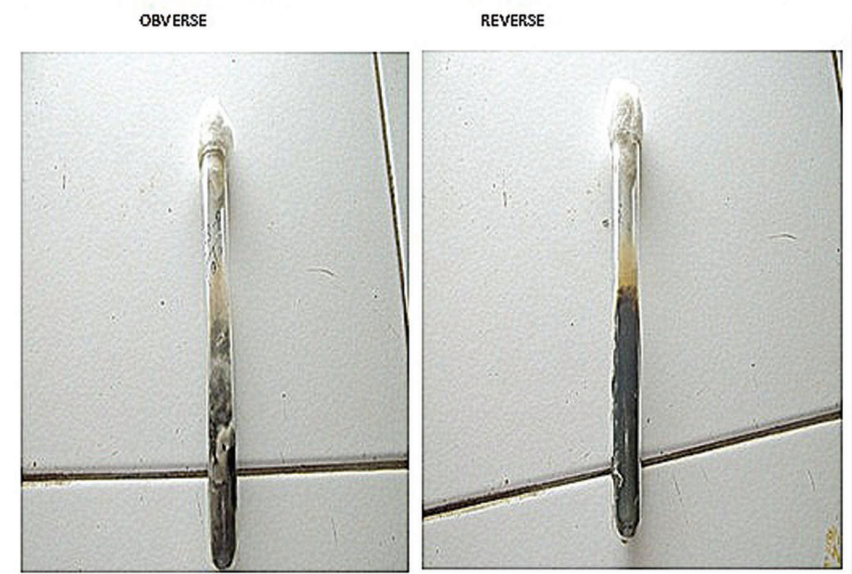

Figure 2. Growth of Exherohilum on SDA slant. 


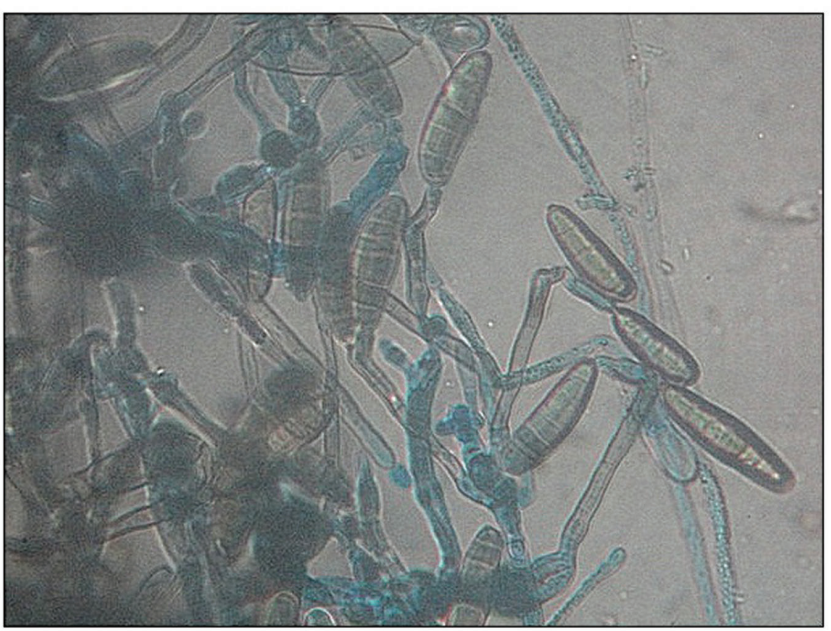

Figure 3. LPCB mount of Exserohilum growth from slide culture showing conidia with protruding hilum.

Outcome appeared to be better than for other mold infections and depended mainly on the underlying diseases [6]. Subcutaneous tissue is a common site for phaeohyphomycosis and is generally thought to occur as a result of traumatic implantation of fungal material from contaminated plants or soil. Corneal infections are due to contamination of traumatic corneal abrasions with asexual fungal spores [3]. A rare case of corneal phaeohyphomycosis due to Exserohilum rostratum following organic trauma was reported from Department of Microbiology, Jawaharlal Institute of Postgraduate Medical Education and Research, Pondicherry, India in April 1996 [7].

Reports of four cases with two types of skin infection, cutaneous and subcutaneous caused by Exserohilum rostratum in immunocompromised men were published by the Department of Dermatology of the National Cheng-Kung University Hospital, Tainan, Taiwan in 1993 [8].

A subcutaneous abscess caused by Exserohilum rostratum was diagnosed in 55 year old healthy woman with a subcutaneous abscess and systemic symptoms of nausea, dizziness and chills following minor trauma to her leg [9]. Another case of phaeohyphomycosis of the nasal sinuses caused by a new species of Exserohilum was reported in a 27 year old man with a 6 year history of allergies developed nasal polyps that occluded his nose [4].

The pathogen is usually an environmental contaminant. Cases of nosocomial fungal infections linked to contaminated care material, especially cloth tape, adhesive tapes or wooden devices were reported in the literature [7]. It is recommended that when Bipolaris or Exserohilum species are isolated from clinical specimens, such isolates should not be carried out simply as contaminants; appropriate studies should be carried out to determine if they play an etiologic role [4].

This is the first diagnosed case of subcutaneous phaeohyphomycosis caused by Exserohilum species reported in HIV positive patient in and around Vijayawada.

In all suspected cases of phaeohyphomycosis the relevant

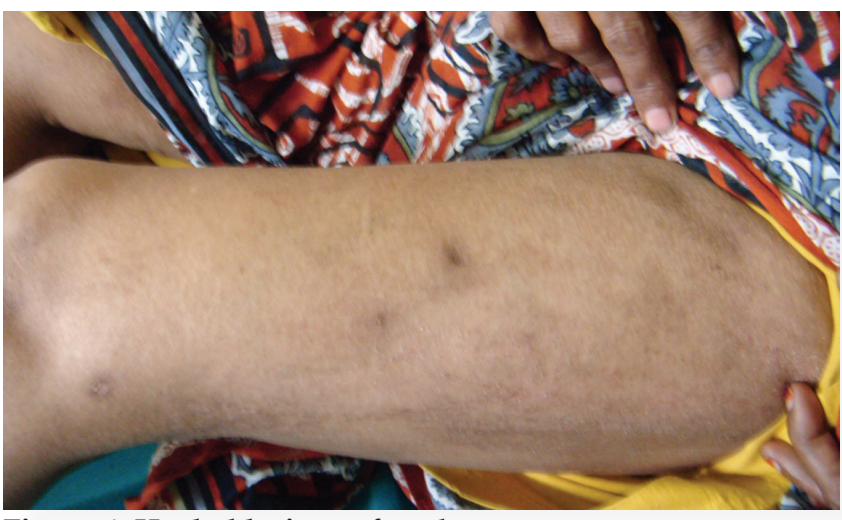

Figure 4. Healed lesions after the treatment. specimens should be sent to the Department of Microbiology \& Department of Histopathology for identification of the causative etiological agent for better patient care and treatment.

We extend our thanks to the Department of Dermatology for their cooperation.

\section{REFERENCES}

1. Lyons JL, Gireesh ED, Trivedi JB, Bell WR, Cettomari D, Smith BR, et al: Fatal Exserohilum Meningitis and Central Nervous System vasculitis after Cervical Epidural Methylprednisolone injection. Ann Intern Med. 2012;157:835-6.

2. Agarwal A, Singh SM: A case of cutaneous phaeohyphomycosis caused by Exserohilum rostratum, it's in vitro sensitivity and review of literature. Mycopathologia. 1995;131:9-12.

3.Coorper Jr CR: Deep phaeohyphomycosis,chapter 35. In: Topley $\&$ Wilson's Microbiology \& Microbial infections. Medical Mycology 10th ed. William G.Merz and Roderick J.Hay, editors. (Hodder Arnold, 338 Easton Road, London NW-1 38 H) 2005: 739-740.

4. Padhye AA, Ajello L, Wiedenand MA, Steinbronn KK: Phaeohyphomycosis of the nasal sinuses caused by a new species of Exserohilum. J Clin Microbiol. 1986;24:245.

5. Therese KL, Madhavan HN: Exserohilum, Chapter 9.In: Molecular Detection of Human Fungal Pathogens, Dongyou Liu, editor (CRC Press) 2011: 83.

6. Adler A, Yaniv I, Samra Z, Yacobovich J, Fisher S, Avrahami G, et al: Exserohilum: an emerging human pathogen. Eur J Clin Microbiol Infect Dis. 2006;25:247-53.

7. Anandi V, George JA, Thomas R, Brahmadathan KN, John TJ: Phaeohyphomycosis of the eye caused by Exserohilum rostratum in India. Mycoses. 1991;34:489-91.

8. Hsu MM, Lee JY: Cutaneous and subcutaneous phaeohyphomycosis caused by Exserohilum rostratum. Arch Dermatol. 1993;28:340-4.

9. Burges GE, Walls CT, Maize JC: Subcutaneous phaeohyphomycosis caused by Exserohilum rostratum in an immunocompetent host. Arch Dermatol. 1987;123:1346-50. 